\title{
23. The effect of Reflexology in patients with Fibromyalgia
}

Sofia Raptopoulou ${ }^{1, *}$, Maria Kouri ${ }^{1}$, Athina Vadalouka ${ }^{1}$, Athanasia Tsaroucha ${ }^{1}$

${ }^{1}$ A' Anesthesiology Department, Pain and Palliative Care Center, Aretaeion Hospital, National and Kapodistrian University of Athens, Athens, Greece. * sofiarapt@gmail.com

Aim: This study is to investigate the effect of Reflexology as an add-on treatment in patients with Fibromyalgia, receiving pregabalin as treatment.

Method: The study was conducted from September 2017 to February 2020.

79 patients ( 74 women and 5 men) with a mean age of 54.6 years were included.

Inclusion criteria:

- Diagnosis of Fibromyalgia $>1$ year

- Duration of pain $>1$ year

- Pain intensity $>6$ on VAS scale

- Not using Reflexology in the past

Results:

- Primary endpoint: Reduction of pain scores more than $30 \%$ in 3 months.

- Secondary endpoints: Functionality (in terms of rigidity and workability) in 3 months.

In all patients, the diagnosis of Fibromyalgia was made by completing the FIRST (Fibromyalgia Rapid Screening Tool) questionnaire (Greek version). The evaluation of the results was done with the pain intensity questionnaire in scale VAS as well as with observation, interviews, diaries.

All patients received Pregabalin medication at a recommended dose of 75-600 mg daily.

44 patients $(56 \%)$ received normal medication. A mean reduction in pain of $>50 \%$ was recorded.

35 patients (44\%) did not reach the maximum dose due to side effects and the drug was stopped at $450 \mathrm{mg}$ daily. These patients received 14, 20-min Reflexology sessions, in 12 weeks, in order to achieve the desired analgesic effect.

There was a reduction of pain $>65 \%$ and a significant improvement in the secondary endpoints of functionality (significantly reduced morning stiffness, increased ability to work).

Conclusions: Reflexology may be beneficial as add-on treatment in patients with Fibromyalgia who are unable to receive the recommended dosages of their medication. 